\title{
Mit der Stadtgesellschaft ins Spiel kommen - Der Fonds „Heimspiel“ der Kulturstiftung des Bundes
}

\section{Lukas Stempel}

\section{Zusammenfassung}

Der Beitrag analysiert das von 2005-2013 von der Kulturstiftung des Bundes realisierte Förderprogramm „Heimspiel“. Zwei Forschungsfragen stehen im Fokus: Welche Häuser und welche Projekte wurden im Rahmen des Fonds „Heimspiel“" gefördert? Und: Wie beschäftigen sich diese mit der Stadt und Stadtgesellschaft? Im Rahmen einer deskriptiv angelegten qualitativen Dokumentenanalyse werden unter anderem Zahlen aus der Deutschen Theaterstatistik und die Kurzbeschreibungen der jeweiligen Stadtprojekte zur Auswertung herangezogen und diskutiert.

\section{Schlüsselwörter}

Bundeskulturstiftung • Heimspiel • Stadtgesellschaft • Partizipation

\section{$1 \quad$ Einleitung}

„Das Theater braucht Durchlässigkeit, Begegnungen mit dem, was außerhalb seiner selbst geschieht, um sich nicht selbst mit der Welt zu verwechseln. Es bedarf dazu Kommunikation und Konzentration. Das bedeutet, es geht bei Partizipation nicht primär darum, kulturelle Angebote verständlicher, attraktiver oder populärer zu kommunizieren, sie besser zu erklären. Es geht in erster Linie darum, wirklich im Kontakt zu sein

\footnotetext{
L. Stempel $(\varangle)$

Institut für Theaterwissenschaft, Ludwig-Maximilians-Universität München, München, Deutschland

E-Mail: Lukas.Stempel@lmu.de
} 
mit denen, für die das Theater da ist. Dazu muss die Schwelle in beide Richtungen überschritten werden: rein und raus“" (Khuon 2019, S. 13).

In seiner Eröffnungsrede zum 4. Europäischen Bürgerbühnenfestival 2019 in Dresden erklärt Ulrich Khuon, Präsident des Deutschen Bühnenvereins, dass partizipative Projekte an den deutschen, öffentlich getragenen Häusern einen hohen Stellenwert haben. Khuon, seit der Spielzeit 2009/2010 Intendant des Deutschen Theaters in Berlin, konstatiert darüber hinaus: Partizipation ermögliche eine andere und neue Form der Auseinandersetzung mit der Stadt und Stadtgesellschaft. Dass eine Vielzahl der öffentlich getragenen Häuser partizipative Projekte im Programm realisieren, offenbart eine Analyse der Spielzeithefte der Saison 2018/2019: 90 der 143 öffentlich getragenen Theater bieten partizipative Formen und Formate im Programm an, dies entspricht rund $63 \%$. Zusätzlich kann festgestellt werden, dass die Relevanz der partizipativen Projekte an den Häusern in den letzten 15 Jahren deutlich gestiegen ist. Exemplarisch zeigt sich dies in Form der Bürgerbühne. 2009 erstmalig als Sparte am Staatsschauspiel in Dresden gegründet, haben in den Folgejahren eine Vielzahl von Theatern ähnliche, vom Titel her gleiche, partizipative Formen in ihre Programme aufgenommen. ${ }^{1}$ Gewiss sind die jeweiligen Bürgerbühnen in der inhaltlichen Ausrichtung und auch organisatorischen Justierung sehr verschieden, dennoch lässt sich konstatieren: Alle Häuser vereinen unter dem Begriff der Bürgerbühne die Arbeit beziehungsweise das Spiel mit nichtprofessionellen Darsteller*innen und dieser partizipativen Form wird im Programm verstärkt Aufmerksamkeit geschenkt. Besonders ein kulturpolitisches Förderprogramm hat die Entwicklung und Stärkung derartiger partizipativer Formen in den Programmen der öffentlich getragenen Theater mitbestimmt: der Fonds „Heimspiel“ der Kulturstiftung des Bundes. ${ }^{2} 2005$ initiierte die Kulturstiftung des Bundes mit diesem Fonds ein Förderprogramm, welches besonders den Austausch zwischen Stadt, Theater und Stadtgesellschaft verstärken sollte.

\footnotetext{
${ }^{1}$ Eine Auswahl der neu gegründeten Bürgerbühnen: seit der Spielzeit 2009/2010 Bürgerbühne am Staatsschauspiel Dresden, seit 2012/2013 Bürgerbühne am Nationaltheater Mannheim, seit 2013/2014 Bürgerbühne am Theater der Altmark, seit 2014/2015 Bürgerbühne an der Burghofbühne Dinslaken und Bürgerbühne am Meininger Staatstheater, seit 2015/2016 Bürgerbühne am Rheinischen Landestheater Neuss, seit 2016/2017 Bürgerbühne am Düsseldorfer Schauspielhaus, Bürgerbühne am Landestheater Schwaben und Bürgerbühne am Theater Lübeck, seit 2018/2019 Bürgerbühne am Hans Otto Theater Potsdam. Teilweise gab es bereits vor der offiziellen Gründung von Bürgerbühnen an den Häusern partizipative Projekte, jedoch nicht zuvorderst unter dem Titel Bürgerbühne.

${ }^{2}$ Die im fortlaufenden Text verwendeten Begriffe „Kulturstiftung des Bundes“ und „Bundeskulturstiftung“ sowie „Fonds Heimspiel“ und „Heimspielfonds“ sind jeweils als bedeutungsgleich zu betrachten.
} 
Obwohl der Fonds bereits im Jahr 2013 ausgelaufen ist, sind die Auswirkungen der realisierten Projekte noch heute im Programm der Häuser zu finden. Besonders der Terminus Stadtprojekt hat sich seitdem als partizipative Theaterform in den Programmen der öffentlich getragenen Theater etabliert. Zusätzlich haben sich in den letzten Jahren bundesweit vermehrt ganze Sparten gegründet, welche die partizipativen Theaterformen bündeln und organisational rahmen. Der vorliegende Beitrag will den kulturpolitischen Akteur ${ }^{3}$ Bundeskulturstiftung und sein Programm „Heimspiel“ näher betrachten. $\mathrm{Zu}$ erforschen gilt es demnach: Welche Häuser und welche Projekte wurden im Rahmen des Fonds „Heimspiel“ gefördert? Und: Wie beschäftigen sich diese mit der Stadt und Stadtgesellschaft?

Im Rahmen einer deskriptiv angelegten qualitativen Dokumentenanalyse werden im Folgenden unter anderem Zahlen aus der Deutschen Theaterstatistik und Kurzbeschreibungen der jeweiligen Stadtprojekte zur Auswertung herangezogen und diskutiert. Der Forschungsstand zum „Heimspielfonds“ ist überraschenderweise von einer gewissen Diffizilität geprägt. Zwar sind Kritiken und Stückbesprechungen in Theater-Fachzeitschriften und Onlineportalen wie ,nachtkritik.de" zu finden, eine differenzierte wissenschaftliche Auseinandersetzung mit dem Forschungsgegenstand ist jedoch nahezu inexistent. ${ }^{4}$ Die Berichterstattung zum „Heimspielfonds“ vonseiten der Kulturstiftung des Bundes beschränkt sich hauptsächlich auf die eigene Webseite.

\section{Partizipation en vogue}

Bevor der Fonds „Heimspiel“ und dessen Struktur näher analysiert wird, ist es essenziell, einen Terminus in den Mittelpunkt der Ausführungen zu platzieren, welcher nahezu durchgehend mit den Stadtprojekten in Kohärenz gesetzt wird: den Begriff der Partizipation. ${ }^{5}$ Besonders in den letzten Jahren hat sich die Auseinandersetzung mit der Begrifflichkeit intensiviert und stark differenziert. ${ }^{6}$

\footnotetext{
${ }^{3}$ Dass die Kulturstiftung des Bundes als kulturpolitischer Akteur begriffen werden kann, konstatieren u. a. Armin Klein (vgl. 2009, S. 137 ff.) in „Kulturpolitik“ und Fabian Leber (2010, S. 133) in „Kulturpolitik aus dem Kanzleramt“.

${ }^{4}$ Im Online-Archiv der Fachzeitschrift „Deutsche Bühne“ lassen sich demnach lediglich acht Artikel finden, die den „Heimspielfonds“ bzw. realisierte Projekte intensiver diskutieren, auf „nachtkritik.de“ sind es sieben Artikel.

${ }^{5}$ Partizipation trägt laut dem Duden folgende Bedeutung: das Teilhaben, Teilnehmen, Beteiligtsein (Duden 2020).

${ }^{6}$ So resultiert Adam Czirak (2014, S. 247) im Rahmen seines Artikels zu den Veränderungspotentialen im Umgang mit dem Begriff der Partizipation: „Nach dem heutigen Stand der Dinge
} 
Dennoch resultiert Christoph Scheurle (2017, S. 19), ,dass der Partizipationsdiskurs in der Theaterwissenschaft noch ganz am Anfang steht“. Zwei Jahre nach dieser Feststellung hat Johannes Kup (2019) mit „, Das Theater der Teilhabe“ eine - auch für die Theaterwissenschaft - umfassende Studie zum Umgang mit dem Begriff der Partizipation veröffentlicht, ausgehend von einer theaterpädagogischen Perspektive. Nach einer ausführlichen Auseinandersetzung mit der Begrifflichkeit Partizipation, unter anderem mit theoretischen und historischen Studien, resultiert er auf die Theaterwissenschaft und deren Verhältnis zum Terminus bezogen:

„Der Begriff der Partizipation bleibt hingegen in der Regel mit sozialen und politischen Fragen verknüpft und bezieht sich im kunst- und theaterwissenschaftlichen Diskurs sowohl auf die Beteiligung von Rezipient*innen als auch auf die Teilnahme nichtprofessioneller Performer*innen oder Spieler*innen“ (Ebd., S. 120).

Kup akzentuiert mit dieser Definition eine Ambivalenz, welche den Begriff der Partizipation prägt: Es muss zwischen Beteiligung und Teilnahme unterschieden werden. Besonders in den Projekten des „Heimspielfonds“ fällt auf, dass die Grenzen zwischen Beteiligten und Teilnehmenden fließend erscheinen. Vermehrt war es Ziel, dass die Projekte nicht nur zur aktiven Teilnahme ermutigen, sondern dass auch das zunächst passive Publikum in den Aufführungsprozess involviert ist. Diese Problematik scheint auch in den gegenwärtigen partizipativen Theaterprojekten nicht gänzlich geklärt. So finden sich in den Programmen immer wieder Formen, in welchen Partizipation als Teilnahme, als Beteiligung oder als ein „Dazwischen“ begriffen werden kann.

droht dem P.-Begriff eine Inflationierung, die zurückzuführen ist auf die oben geschilderte, massive Veränderung der Zuschauer- bzw. Betrachterrolle in allen Bereichen der Kunstpraxis seit den 1960er Jahren“. Dass der Terminus in der wissenschaftlichen Diskussion äußerst unterschiedlich Verwendung findet, zeigen u. a. die folgenden Publikationen: Azadeh Sharifi (2011) verhandelt in „Theater für Alle?“ im Rahmen von kulturpolitischen Perspektiven und einer interkulturellen Öffnung die Partizipation von Postmigrant*innen im Theater, Adam Czirak (2012) in „Partizipation der Blicke“ die sich verändernden Sehgewohnheiten im Zuschauerraum und die Autoren Wolfgang Schneider und Anna Eitzeroth (2017) im Sammelband „Partizipation als Programm“ (neue) Wege im Programm durch Partizipation im Theater für Kinder und Jugendliche. 


\section{Der Fonds „,Heimspiel“}

2005 wurde von der Kulturstiftung des Bundes der „Heimspielfonds“ initiiert, wodurch 62 Stadtprojekte antragsbasiert bis 2013 realisiert werden konnten. ${ }^{7}$ Folgende Ziele wurden damit von der Bundeskulturstiftung proklamiert:

„Gefördert wurden Theaterprojekte, die sich mit der urbanen und sozialen Realität der Stadt auseinandersetzen und ein neues Publikum für das (Stadt-)Theater gewinnen sollten. Das Theater kann für das Selbstverständnis einer Stadt eine zentrale Rolle spielen. Es ist ein öffentlicher Ort, an dem sich Menschen versammeln, an dem sie sich mit sich selbst und der Gesellschaft auseinandersetzen. Dennoch müssen alle öffentlichen Theater um die Akzeptanz des Publikums und nicht zuletzt um ihre finanzielle Ausstattung kämpfen. Was bedeutet es heute Theater für eine Stadt zu machen? Wie kann das Theater zu einem Ort werden, der sich den drängenden Problemen der Gegenwart stellt? Wie kann es ein neues Publikum gewinnen und gleichzeitig seinem Stammpublikum ungewohnte Sichtweisen präsentieren?“ (Kulturstiftung des Bundes 2020).

Als Ausgangspunkt des Förderfonds gilt also die Annahme, dass die Daseinsberechtigung öffentlich getragener Häuser in der Stadt kein evidentes Gut (mehr) ist. In erster Linie wird vor allem die finanzielle Lage der Theater thematisiert, die nicht gesichert scheint. ${ }^{8}$ Die Kulturstiftung des Bundes wollte demnach mit dem Fonds vor allem erreichen, dass sich Theatermacher*innen einerseits mit aktuellen Themen der Stadtgesellschaft ${ }^{9}$ auseinandersetzen. Andererseits sollten durch diesen Austausch und die Schaffung innovativer künstlerischer Formen neue Besucher*innen für das Theater gewonnen werden. Ein Dialog zwischen

\footnotetext{
${ }^{7}$ Zudem wurde das Projekt „Bunny Hill 2“ an den Münchner Kammerspielen 2006 ergänzend gefördert, sowie zwei Werkstätten 2006 in München und 2007 in Hamburg. Darüber hinaus fand zusätzlich 2011 eine mehrtägige Veranstaltung statt, die u. a. eine Reflexion über das Förderprogramm ermöglichte. Bestehend aus einem Symposium, einem Festival, Workshops und einem begehbaren Archiv sollte der künstlerische und wissenschaftliche Austausch über die Projekte und die Ziele des Fonds intensiviert werden.

${ }^{8}$ Um das Jahr 2005 bzw. besonders die Jahre davor wurden Diskurse geführt, die konkret auch die teilweise prekären finanziellen Ausstattungen der Theater in den Mittelpunkt rückten. Die Fachzeitschrift „Deutsche Bühne“ widmet bspw. im Heft 4 im Jahr 2003 der Thematik einen Schwerpunkt unter dem Titel „Vor dem Flächenbrand? Die Theater und die Finanzkrise“ und die Kulturpolitische Gesellschaft veröffentlicht ein Jahr später ihr umfangreiches Jahrbuch mit dem Titel „Thema: Theaterdebatte“ (Wagner 2004) mit mehreren Artikeln zur finanziellen Situation der öffentlich getragenen Theater. Demnach ist es kaum verwunderlich, dass die Kulturstiftung des Bundes diesen Diskurs aufgreift.

${ }^{9}$ Den Begriff Stadtgesellschaft definiert Birgit Mandel facettenreich in Ihrer Einführung in das Teilkapitel (Kap. 17 dieses Bandes).
} 
den Bürger*innen der Stadt und dem jeweiligen (Stadt-)Theater sollte neue Beziehungsrelationen etablieren. Zudem wurde von den Antragssteller*innen erwartet, dass vor der Projektantragsphase bereits umfangreich recherchiert wurde und konkret bestimmte Gruppierungen der Stadt erforscht wurden. ${ }^{10}$ In elf Jurysitzungen wurden die eingereichten Projektanträge besprochen, respektive die Förderzusagen erteilt. Die Jury bestand stets aus drei Personen. ${ }^{11} \mathrm{Zu}$ den Rahmendaten: 47 Theater $^{12}$ aus 42 Städten wurden im Rahmen des Fonds „Heimspiel“ der Kulturstiftung des Bundes gefördert. ${ }^{13}$ Wie Abb. 1 verdeutlicht, befinden sich über zwei Drittel (72,34 \%) der am „Heimspiel“ beteiligten Häuser in Städten mit über 100.000 Einwohner*innen, fast ein Drittel (31,91 \%) sogar in Städten mit über 500.000 Einwohner*innen. Lediglich sieben der Städte mit Theatern $(14,89 \%)$ haben hingegen unter 50.000 Einwohner*innen. ${ }^{14}$

\footnotetext{
${ }^{10}$ Es fällt auf, dass sich die künstlerische Ausrichtung des Fonds an gegenwärtigen Tendenzen orientierte, die seit dem Jahr 2000 deutlich vermehrt aufgetreten sind. Es handelt sich hier vor allem um partizipative Rechercheprojekte bzw. allgemein um Theaterformen, welche Laien und Expert*innen des Alltags zum Mitmachen ermächtigten. Zu nennen sind hier u. a. die Arbeiten des Kollektivs Rimini Protokoll (Dreysse und Malzacher 2007). Im Rahmen einer Analyse von Artikeln der Zeitschrift „Deutsche Bühne“ zeigt sich zudem, dass die Auseinandersetzung mit der Thematik „Partizipation“ und „Laien auf der Bühne“ ab den 2000er Jahren kontinuierlich an Wertigkeit gewonnen hat. So lassen sich 200215 Artikel, 20039 Artikel, 200414 Artikel, 200542 Artikel und 200641 Artikel in den Ausgaben der „Deutschen Bühne“ identifizieren, die sich konkret mit partizipativen Formen auseinandersetzen.

${ }^{11}$ Der ersten Jurysitzung wohnten Thomas Bockelmann, Matthias Lilienthal und Peter Kastenmüller bei, Jurysitzung zwei bis fünf Thomas Bockelmann, Nuran Calis und Matthias Lilienthal, Jurysitzung sechs Nuran Calis, Matthias Lilienthal und Tobias Wellemeyer und Jurysitzung sieben bis elf Olaf Kröck, Tobias Rausch und Tobias Wellemeyer. Es zeigt sich deutlich, dass versucht wurde über einen bestimmten Zeitraum auf eine gewisse Konstanz in der Jury zu setzen. Zudem waren neben einem Intendanten stets ein Regisseur (und Autor) sowie Dramaturg bzw. künstlerischer Leiter Teil der Begutachtung.

${ }^{12}$ Das Staatstheater Stuttgart und das Staatstheater Hannover sind u. a. in der Deutschen Theaterstatistik als jeweils eine organisatorische Einheit geführt, d. h. Schauspiel, Junges Schauspiel und Oper sind organisational vereint. Im Rahmen des Programms sind die jeweiligen Sparten der Häuser jedoch klar separat zu betrachten. Die Stadtprojekte der Theater können demnach konkret Sparten zugeordnet werden; so werden die Häuser auch in den vorliegenden statistischen Auswertungen separat betrachtet.

${ }^{13}$ An mehreren Häusern wurden zwei bzw. drei Projekte im „Heimspielfonds“ realisiert. Dazu gehören das Anhaltische Theater Dessau (2x), das Theater Dortmund (2x), das Schauspiel Frankfurt (2x), das Theater Freiburg (2x), das Theater Vorpommern in Greifswald (2x), das Thalia Theater Halle (2x), das Staatstheater Hannover - Schauspiel (2x), das Theaterhaus Jena (2x), das Puppentheater Magdeburg (3x), die Kammerspiele München (2x), das Theater Oberhausen (3x) und das Thüringer Landestheater Rudolstadt (2x).

${ }^{14}$ Die Stadttypen richten sich nach der Einteilung des Bundesinstituts für Bau-, Stadt- und Raumforschung (Stand: 29.01.2020). Landgemeinde unter 5000 Einwohner*innen, Kleine
} 


\begin{tabular}{|l|l|l|}
\hline Stadttyp & Theater & in Prozent \\
\hline Landgemeinde & 1 & $02,13 \%$ \\
\hline Kleine Kleinstadt & 0 & $00,00 \%$ \\
\hline Größere Kleinstadt & 0 & $00,00 \%$ \\
\hline Kleinere Mittelstadt & 6 & $12,77 \%$ \\
\hline Größere Mittelstadt & 6 & $12,77 \%$ \\
\hline Kleinere Großstadt & 19 & $40,43 \%$ \\
\hline Große Großstadt & 15 & $31,91 \%$ \\
\hline
\end{tabular}

Abb. 1 Geförderte Theater nach Stadttypen, Fonds „Heimspiel“ der Kulturstiftung des Bundes. (Quelle: Eigene Berechnungen. (C) Lukas Stempel, 2020)

Zudem liegen über $60 \%$ der am „Heimspielfonds“ beteiligten Theater $(61,70 \%)$ in Westdeutschland, 18 Theater $(38,30 \%)$ in Ostdeutschland. In 13 von 16 Bundesländern wurden Projekte realisiert. Baden-Württemberg und Nordrhein-Westfalen haben mit zehn $(21,28 \%)$, respektive sieben $(14,89 \%)$, die höchste Anzahl an geförderten Stadtprojekten. In Rheinland-Pfalz, im Saarland und in Schleswig-Holstein wurden keine Projekte umgesetzt. Im Rahmen der Betrachtung von Abb. 2 fällt besonders auf, dass im eher kleineren Bundesland Sachsen-Anhalt sechs Projekte $(12,77 \%)$ realisiert wurden. ${ }^{15}$

Deutlich mehr als die Hälfte der am Fonds „Heimspiel“ beteiligten Theater sind Stadttheater $(59,57 \%)$, etwas mehr als ein Viertel Staatstheater $(27,66 \%)$ und sechs Häuser $(12,77 \%)$ sind als Landestheater organisiert. Um einen diverseren Überblick zu erhalten, wie die im „Heimspielfonds“ der Kulturstiftung des Bundes geförderten Theater organisational und strukturell aufgestellt sind, lohnt sich ein Blick in die Theaterstatistik des Deutschen Bühnenvereins. ${ }^{16}$ Abb. 3 zeigt in 21 Kategorien die Durchschnittswerte sowie die jeweiligen Minima und

Kleinstadt unter 10.000, Größere Kleinstadt unter 20.000, Kleinere Mittelstadt unter 50.000, Größere Mittelstadt unter 100.000, Kleinere Großstadt unter 500.000 und Großstadt mind. 500.000 Einwohner*innen.

${ }^{15}$ Sachsen-Anhalt ist im bundesdeutschen Vergleich die Einwohner*innenzahl betreffend auf Platz 11 von 16 (Stand: 31.12.2018). Mit acht öffentlich getragenen Häusern liegt SachsenAnhalt in der Theaterstatistik 2016/2017 - die Anzahl der Häuser je Bundesland betreffend - auf Platz 7 von 16.

${ }^{16}$ Nicht alle im „Heimspielfonds“ geförderten Theater können in die Untersuchung einbezogen werden, da keine umfänglichen Statistiken in der Theaterstatistik aufgeführt sind. Das Theaterhaus Jena wird als Privattheater aufgeführt, das Theater Paderborn - Westfälische Kammerspiele und das Theater Lindenhof Burladingen/Melchingen ebenso. 


\begin{tabular}{|l|l|l|}
\hline Bundesland & Theater & in Prozent \\
\hline Baden-Württemberg & 10 & $21,28 \%$ \\
\hline Bayern & 2 & $04,26 \%$ \\
\hline Berlin & 3 & $06,38 \%$ \\
\hline Brandenburg & 2 & $04,26 \%$ \\
\hline Bremen & 2 & $04,26 \%$ \\
\hline Hamburg & 1 & $02,13 \%$ \\
\hline Hessen & 2 & $04,26 \%$ \\
\hline Mecklenburg-Vorpommern & 1 & $02,13 \%$ \\
\hline Niedersachsen & 4 & $08,51 \%$ \\
\hline Nordrhein-Westfalen & 7 & $14,89 \%$ \\
\hline Rheinland-Pfalz & 0 & $00,00 \%$ \\
\hline Saarland & 0 & $00,00 \%$ \\
\hline Sachsen & 4 & $08,51 \%$ \\
\hline Sachsen-Anhalt & 6 & $12,77 \%$ \\
\hline Schleswig-Holstein & 0 & $00,00 \%$ \\
\hline Thüringen & 3 & $06,38 \%$ \\
\hline
\end{tabular}

Abb. 2 Geförderte Theater nach Bundesland, Fonds „Heimspiel“ der Kulturstiftung des Bundes. (Quelle: Eigene Berechnungen. (C) Lukas Stempel 2020)

Maxima. ${ }^{17}$ Haben die Durchschnittswerte einen eher instruktiven Charakter, sind im Kontrast dazu die Spalten Minimum und Maximum äußerst aufschlussreich. So zeigt sich nahezu in allen Kategorien, wie divergent und divers sich die Struktur der am Förderfonds beteiligten Theater darstellt.

Besonders große Kontraste können in den Bereichen Vorstellungen (Min. 131, Max. 1059), Besucher*innen Gesamt (Min. 6127, Max. 548.347), Personal Gesamt (Min. 26, Max. 1297), Einnahmen Insgesamt (Min. 1442, Max. 92.502)

\footnotetext{
${ }^{17}$ Auf die Errechnung des Median und der Standardabweichung (die Streuung der Werte) wurde bewusst verzichtet; die Ergebnisse einer derartigen Auswertung werden für die Argumentationsstruktur nicht als zentral erachtet. Da die Förderdauer sich über mehrere Jahre hinweg gezogen hat, sind die Daten aus der Theaterstatistik für das jeweilige Theater aus dem Jahr entnommen, in welchem (erstmalig) das Theater in der Jurysitzung Erwähnung findet bzw. den Zuschlag zur Realisierung eines Projekts erhält. Die Grundgesamtheit (n) kann variieren, da einige Häuser in der Theaterstatistik im Rahmen der jeweiligen Kategorien keine Angaben machen.
} 


\begin{tabular}{|c|c|c|c|c|}
\hline Kategorie & Mittelwert & Min. & Max. & $\mathrm{n}$ \\
\hline $\begin{array}{l}\text { Plätze (auf } 1.000 \\
\text { Einwohner*innen) }\end{array}$ & 7 & 2 & 31 & 44 \\
\hline Anzahl der Spielstätten & 7 & 2 & 22 & 44 \\
\hline Anzahl der Vorstellungen & 528 & 131 & 1.059 & 43 \\
\hline Sonstige Veranstaltungen & 80 & 2 & 378 & 42 \\
\hline $\begin{array}{l}\text { Theaternahes } \\
\text { Rahmenprogramm }\end{array}$ & 127 & 9 & 846 & 30 \\
\hline Veranstaltungen Gesamt & 607 & 159 & 1.252 & 44 \\
\hline $\begin{array}{l}\text { Besucher*innen } \\
\text { Sonstige Veranstaltungen }\end{array}$ & 16.923 & 473 & 102.870 & 42 \\
\hline $\begin{array}{l}\text { Besucher*innen } \\
\text { Theaternahes } \\
\text { Rahmenprogramm }\end{array}$ & 7442 & 400 & 31.440 & 29 \\
\hline Besucher*innen Gesamt & 181.258 & 6.127 & 548.347 & 44 \\
\hline $\begin{array}{l}\text { Nicht Darstellendes } \\
\text { Künstlerisches Personal }\end{array}$ & 37 & 3 & 133 & 41 \\
\hline Künstlerisches Personal & 150 & 9 & 508 & 44 \\
\hline Personal Gesamt & 352 & 26 & 1.297 & 44 \\
\hline $\begin{array}{l}\text { Betriebseinnahmen (in } \\
1.000 \text { Euro) }\end{array}$ & 3.677 & 93 & 18.581 & 44 \\
\hline $\begin{array}{l}\text { Zuweisungen und } \\
\text { Zuschüsse (in } 1.000 \text { Euro) }\end{array}$ & 19.756 & 1.040 & 76.635 & 44 \\
\hline $\begin{array}{l}\text { Einnahmen Insgesamt (in } \\
\text { 1.000 Euro) }\end{array}$ & 23.013 & 1.442 & 92.502 & 44 \\
\hline $\begin{array}{l}\text { Ausgaben Personal (in } \\
\text { 1.000 Euro) }\end{array}$ & 17.830 & 1.082 & 71.921 & 44 \\
\hline $\begin{array}{l}\text { Ausgaben Betrieb (in } \\
1.000 \text { Euro) }\end{array}$ & 4.972 & 354 & 19.294 & 44 \\
\hline $\begin{array}{l}\text { Ausgaben Insgesamt (in } \\
1.000 \text { Euro) }\end{array}$ & 23.913 & 1.442 & 94.340 & 44 \\
\hline $\begin{array}{l}\text { Einspielergebnis (in } \\
\text { Prozent) }\end{array}$ & 15 & 5 & 29 & 44 \\
\hline $\begin{array}{l}\text { Betriebszuschuss (pro } \\
\text { Besucher*in in Euro) }\end{array}$ & 109 & 41 & 238 & 44 \\
\hline $\begin{array}{l}\text { Durchschn. Erlös (pro } \\
\text { Besucher*in in Euro) }\end{array}$ & 18 & 4 & 37 & 44 \\
\hline
\end{tabular}

Abb. 3 Verschiedene Charakteristika der geförderten Theater, Fonds „Heimspiel“ der Kulturstiftung des Bundes. (Quelle: Eigene Berechnungen. (C) Lukas Stempel, 2020)

und Betriebszuschuss (Min. 41, Max. 238) eruiert werden. Es kann also konstatiert werden, dass mit dem „Heimspielfonds“ ein differenziertes Spektrum an Theatern erreicht wurde, beziehungsweise dass sich Häuser mit unterschiedlichsten Charakteristika für den Förderfonds interessierten. 62 Kurzbeschreibungen der im „Heimspielfonds“ realisierten Projekte sind auf der Webseite der Kulturstiftung des Bundes zu lokalisieren. Zu Beginn einer inhaltlichen Auseinandersetzung mit den jeweiligen Projektinhalten ist zunächst eine einfache Analyse von Worthäufigkeiten von Nutzen. 


\begin{tabular}{|l|l|l|}
\hline Wort & Häufigkeit & in Prozent \\
\hline theater & 54 & 0,65 \\
\hline stadt & 40 & 0,48 \\
\hline projekt & 38 & 0,45 \\
\hline menschen & 32 & 0,38 \\
\hline gemeinsam & 20 & 0,24 \\
\hline bühne & 18 & 0,22 \\
\hline jugendlichen & 18 & 0,22 \\
\hline heute & 17 & 0,20 \\
\hline leben & 17 & 0,20 \\
\hline ort & 16 & 0,19 \\
\hline material & 14 & 0,17 \\
\hline arbeit & 13 & 0,16 \\
\hline jugendliche & 13 & 0,16 \\
\hline armut & 12 & 0,14 \\
\hline einwohner & 11 & 0,13 \\
\hline recherche & 11 & 0,13 \\
\hline zusammen & 11 & 0,13 \\
\hline inszenierung & 10 & 0,12 \\
\hline künstlerischen & 10 & 0,12 \\
\hline migranten & 10 & 0,12 \\
\hline neuen & 10 & 0,12 \\
\hline region & 10 & 0,12 \\
\hline thema & 10 & 0,12 \\
\hline
\end{tabular}

Abb. 4 Worthäufigkeiten in den Projektbeschreibungen, Fonds „Heimspiel“ der Kulturstiftung des Bundes. (Quelle: Eigene Berechnungen. (C) Lukas Stempel, 2020)

Abb. 4 zeigt in erster Linie, dass die Wörter „Theater“, „Stadt“ und „Projekt" erwartungsgemäß am häufigsten genannt werden. ${ }^{18}$ Darüber hinaus lassen sich Begriffe wie „gemeinsam“, „heute“, „Leben“, „Recherche“, ,zusammen“,

\footnotetext{
${ }^{18}$ Insgesamt konnten 8362 Wörter (incl. Mehrfachzählung) bzw. 2949 Wörter (nur Einfachzählung) identifiziert werden. Die drei am häufigsten genannten Wörter sind ,und“ (361 Nennungen, 4,32 \%), „die“ (334 Nennungen, 3,99 \%) und „der“ (266 Nennungen, $3,18 \%)$. Im Rahmen der vorliegenden Analyse wurde eine Selektion vorgenommen, d. h. Wörter mit geringem Bedeutungswert (bspw. ,ein“, ,,im“oder „,dem“) wurden von der Betrachtung ausgeschlossen. Die Analyse wurde mit der Software MAXQDA durchgeführt.
} 
„,neuen“ oder „Region“ ermitteln, welche sich nah an den Zielen des „Heimspielfonds" orientieren, das heißt mit hoher Wahrscheinlichkeit aus der Antragsprosa heraus resultieren. Durchaus können jedoch auch Termini entdeckt werden, welche erste Rückschlüsse auf die inhaltliche Ausrichtung der jeweiligen Projekte ermöglichen. So sind die Worte „Jugendliche/n“, „Arbeit“, „Armut“ und „Migranten" ein erstes Indiz dafür, welche inhaltlichen Schwerpunkte die Theater im Rahmen der Stadtprojekte setzten. Diese quantitativen Ergebnisse erfordern durch eine qualitative Betrachtung eine Konkretisierung.

\section{$4 \quad$ Wer, wie, was - wieso, weshalb, warum?}

Bildungsmisere, Rassismus, Prostitution, Jugendkriminalität, Atomkraft, Überalterung - ein Blick in die Themenvielfalt der im „Heimspielfonds“ realisierten Projekte offenbart, dass sich die Theater mit unterschiedlichsten Fragestellungen auseinandergesetzt haben. Um die inhaltliche Analyse zu strukturieren, sollen die Projekte im Folgenden im Rahmen der W-Fragen-Methode (wer, was, wann, wo, warum, wie und wozu) betrachtet werden ${ }^{19}$, und dies aus dem Blickwinkel der jeweiligen Projektskizzen:

\section{Wer wirkte im Rahmen der Projekte mit?}

Neben den Theaterschaffenden wurden gezielt bestimmte Milieus und Gesellschafts- bzw. Berufsgruppen im Kontext der Projektarbeit angesprochen: u. a. Migrant*innen, Hooligans, Gastarbeiter*innen, Senior*innen, Polizist*innen, Bundeswehrsoldat*innen, Arbeitslose, Flüchtlinge - die Aufzählung lässt sich noch weiterführen. Oft waren die jeweiligen Gruppen in den Produktionsund/oder Schaffungsprozess des Projekts direkt eingebunden und nahmen die Rolle eines Experten bzw. einer Expertin ein. Schlussendlich wirkten einige der Akteur*innen dann auch im Rahmen von Produktionen auf der Bühne mit. Es ist klar ersichtlich, dass die Projekte vermehrt an Kinder und Jugendliche adressiert waren. Die Partizipation erfolgte also aktiv (teilnehmend) und/oder passiv (beteiligt).

\section{Was wurde im Rahmen der Projekte verwirklicht?}

Wie in Abb. 5 ersichtlich, lassen sich sechs Themenfelder eruieren, die verstärkt in den Kurzbeschreibungen der Projekte genannt werden:

\footnotetext{
${ }^{19}$ Auf statistische Daten und Erhebungen wird an dieser Stelle und im Folgenden bewusst verzichtet.
} 
Abb. 5 Themenfelder der Projektbeschreibungen, Fonds „Heimspiel“ der Kulturstiftung des Bundes. (Quelle: Eigene Darstellung. (C) Lukas Stempel, 2020)

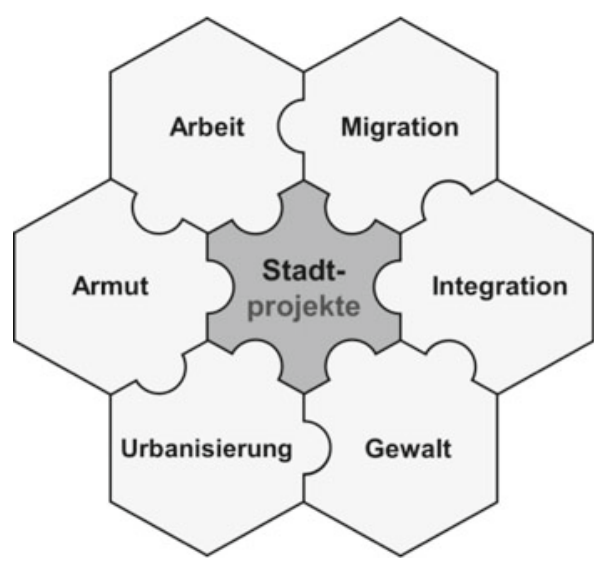

Im Kontext des Feldes Arbeit wird vermehrt die Problematik der Arbeitslosigkeit verhandelt. Die Thematik Migration steht sehr oft im Vordergrund, daran anschließend besonders der Stellenwert der Integration, hier aber nicht nur auf Migrant*innen bezogen, sondern beispielsweise auch auf Menschen mit Behinderung. Einige Projekte befassen sich konkret mit dem Problemfeld Gewalt. Die Stadtentwicklung und mögliche Utopien für eine Stadt der Zukunft - und ein Zusammenleben - finden sich im Bereich Urbanisierung wieder. Auch der Aspekt der Armut steht im Zentrum diverser Projekte. Obwohl im Rahmen der Ziele des Förderfonds indirekt angesprochen, beschäftigt sich lediglich ein Projekt konkret mit der Zukunft des Theaters bzw. entwickelt Ideen für eine Novellierung der Theaterstrukturen.

Wann wurden die Projekte realisiert?

Der Zeitraum des Gesamtprojekts wurde bereits erwähnt. Die Häuser selbst realisierten nach der Zusage der Förderung in ganz verschiedenen Zeitspannen die jeweiligen Projekte.

Wo haben die Projekte stattgefunden?

Im Rahmen der Ziele des Fonds formulierte die Bundeskulturstiftung den Wunsch, dass die Projekte (möglichst) auf der „Großen Bühne“ gezeigt werden. Nahezu alle Theater sind dieser Bedingung nachgekommen. Oft wurde der Abschluss des Projekts jedoch verbunden mit einer außerhalb des Theaters stattfindenden Aktion. Vermehrt wurden „Theatre Walks“ realisiert und/oder Stadtteilrundgänge an einem für das Projekt und die Handlung relevanten Ort. 
Warum wurden die Projekte durchgeführt?

Ein Rückblick auf die von der Kulturstiftung des Bundes für den „Heimspielfonds" definierten Ziele hilft, mögliche Motive der jeweiligen Projekte konkreter zu fassen. Zwei Hauptziele konnten in der vorherigen Argumentation bereits erläutert werden: zum einen die Auseinandersetzung mit Themen der Stadt und zum anderen die Gewinnung neuer Publika. Das erste Ziel ist im Hinblick auf die Kurzbeschreibungen der jeweiligen Projekte fortlaufend erfüllt. Es fällt allerdings auf, dass die Theater sich vor allem in ihren Projekten mit Problemen bzw. direkten Herausforderungen ihrer spezifischen Stadt beschäftigen und diese zum Gegenstand ihrer Recherchen und künstlerischen Arbeit machen. Zusätzlich sind die Theater mit Akteur*innen der Stadt in Austausch getreten und haben diese fast durchgehend in den künstlerischen Produktions-, Schaffungs- und Aufführungsprozess eingebunden. Ob schlussendlich neue Publika durch die Projekte angesprochen wurden, kann nicht verifiziert werden, auch da dazu keine Studien vonseiten der Theater oder der Bundeskulturstiftung vorliegen. Zudem fällt auf, dass die Häuser mit ihren Projekten den Dialog suchen, hin zur Stadtgesellschaft und dies multiperspektivisch. Die forcierten Dialoge können demnach ganz unterschiedliche Formen und Charakteristika annehmen: interkulturell, intergenerativ, interreligiös, integrativ und/oder innerstädtisch. Vereinzelt wird auch ein künstlerischer Austausch angesprochen, d. h. die künstlerischen Akteur*innen der Häuser wollen im Rahmen ihrer Arbeit beziehungsweise durch diese mit den Bürger*innen in den Dialog treten.

Wie wurden die Projekte umgesetzt?

Alle Projekte basieren auf realisierten Recherchen in der Stadt, unter anderem da dies als ein Förderkriterium der Bundeskulturstiftung vorgegeben war. Die Formenvielfalt ist stark ausgeprägt: sie reicht von szenischer Intervention, Kulissenschau, HipHop-Oper über Massentheater, lebendigen Geschichtsunterricht, Straßenoper und Talentgala. Eine Einteilung des jeweiligen Projekts in eine bestimmte Sparte ist demnach eher schwer zu realisieren. Es lässt sich lediglich resümieren, dass nahezu alle gängigen Sparten (Schauspiel, Oper, Tanz und Figurentheater) vertreten sind, ein Großteil lässt sich dem (erweiterten) Begriff des Sprechtheaters zuordnen. 


\section{$5 \quad$ Fazit und Ausblick - Stadtgesellschaft(en) "Heimspiele" ermöglichen}

Die Analysen zum „Heimspielfonds“ haben Folgendes gezeigt:

- Der Fonds „Heimspiel“ hat aus einer strukturellen Perspektive heraus betrachtet ein äußerst heterogenes Teilnehmerfeld.

- Die in den Projekten verhandelten Themen verfügen nahezu ausschließlich über einen problemzentrierten Ausgangspunkt. Das heißt: Es werden in den Projekten vorwiegend städtische und gesellschaftspolitische Herausforderungen diskutiert und reflektiert. Im Rahmen der künstlerischen Arbeit sollen dann Lösungswege und Veränderungspotentiale entwickelt werden.

- Die nichtprofessionellen Akteur*innen wurden entweder passiv beteiligend oder aktiv teilnehmend in die Projekte integriert. Teilweise werden unterschiedliche Formen angeboten, die beide Partizipationsstile ermöglichen.

- Im Fokus der Projekte steht vorwiegend die künstlerische Entwicklung innovativer Theaterformen und das „Ausprobieren“ neuer Ästhetiken. Nur ein Projekt beschäftigt sich konkret mit der Zukunft des Theaters und möglichen Veränderungsdynamiken. ${ }^{20}$

„Ein Stadt- oder Staatstheater liefert im Grunde genommen Abend für Abend ein „Heimspiel“ auf bekannter Bühne, allein die „Fan-Zahlen“ lassen zu wünschen übrig. Und obwohl ein Theatergebäude geografisch meist im Zentrum einer Stadt angesiedelt ist, scheint es im Bewusstsein der Stadtgesellschaft eher einen Platz am „Rande des Spielfelds“"zu haben“ (o. V. 2020).

In allen Projekten stehen die Bürger*innen der Stadt, also die Stadtgesellschaft beziehungsweise Teile davon im Mittelpunkt der theaterpraktischen Arbeit. Der Platz am „Rande des Spielfelds“ sollte im Rahmen des „Heimspielfonds“ einem Platz ,auf dem Spielfeld“ weichen. Die Stadtgesellschaft aktiv einbeziehen, zum

\footnotetext{
${ }^{20}$ Hortensia Völckers, Vorstand und Künstlerische Direktorin der Kulturstiftung des Bundes, erläutert in einem Interview 2007 noch einmal sehr klar die Ziele des Förderfonds: „Wir unterstützen mit dem ,Heimspiel-Fonds ‘ ein genuines Interesse der Theater, ihre Rolle in der Stadt unter heutigen Bedingungen zu behaupten. [...] Aber bei der Auswahl der Projekte aus den vielen Anträgen achtet die Jury sehr darauf, dass ein hoher Anspruch an die künstlerische Qualität erkennbar ist. [...] Der ,Heimspiel-Fonds " versteht sich vor allem als Impulsgeber“ (Völckers 2007). Diese Ziele verdeutlichen nochmals, dass im Vordergrund die künstlerische Projektförderung stand.
} 
Mitmachen animieren und besonders Außenseiter zu Wort kommen lassen. ${ }^{21}$ Haben sich diese von der Kulturstiftung des Bundes geförderten Maßnahmen verstetigt? Eine allumfassende Antwort auf diese Frage kann nicht gegeben werden. Wird der Fokus auf die im „Heimspielfonds“ geförderten Theater gerichtet, fällt vereinzelt auf, dass durch die finanzielle Projektförderung der Kulturstiftung des Bundes durchaus künstlerische Transformationsprozesse begünstigt und unterstützt wurden. ${ }^{22}$ Exemplarisch zeigt sich dies beispielsweise an der Bürgerbühne in Dresden. Im Rahmen der ersten Spielzeit der 2009/2010 neu gegründeten Sparte am Staatsschauspiel wurde das Stadtprojekt „Alles Auf Anfang!“ mit Geldern des „Heimspielfonds“ realisiert. Die Bürgerbühne in Dresden hat sich seitdem in den letzten zehn Jahren etabliert, ist als Teil des Angebots im Programm am Staatsschauspiel fest verankert und hat schlussendlich sogar Intendanzwechsel überdauert: „Seit ihrer Gründung stellt die Bürger:Bühne in Dresden der Stadtgesellschaft einen alternativen Begegnungs- und Gesprächsraum zur Verfügung. Sie verschafft Gehör und Austausch für differenzierte Diskurse und leise Stimmen“" (Staatsschauspiel Dresden 2020). Ähnliche Entwicklungen lassen sich auch am Badischen Staatstheater Karlsruhe beobachten. In der Spielzeit 2011/2012 verwirklichte das Staatstheater das Stadtprojekt „Der Gastfreund/Die Argonauten“. In derselben Spielzeit wurde die Sparte Volkstheater gegründet, welche sich auch heute noch die Frage stellt: „Besitzt das Theater in einer immer diverseren Gesellschaft die Macht, Veränderung anzustoßen und in die Stadtgesellschaft hineinzuwirken?" (Badisches Staatstheater Karlsruhe 2020). Und in Freiburg musste aufgrund einer politischen Legitimationskrise zum Start der Intendanz von Barbara Mundel 2006 die Frage zum „Verhältnis von Stadtgesellschaft und Theater als Kunstform“ neu gestellt werden (Hasselberg 2014). In diesem Kontext wurde das Stadtprojekt „Orbit“ entwickelt und umgesetzt (vgl. Mundel und Mackert 2007). Trotz diverser Veränderungen auf Leitungsebene und einer Neuausrichtung des Hauses seit 2017 existiert beispielsweise das im Rahmen der von Barbara Mundel bewirkten Öffnung zur Stadt entstandene, partizipative „Theaterlabor School of Life and Dance“ auch gegenwärtig.

\footnotetext{
${ }^{21}$ Berit Schuck, künstlerische Leiterin des Festivals „HEIMSPIEL“ von 2011 erklärt in einem Interview zum „Heimspielfonds“: „Es ist durchaus eine Aufgabe der Stadttheater, dass dort Menschen zu Wort kommen, die sonst nicht gehört werden“ (Baur 2011b).

${ }^{22}$ Weiterhin konstatiert Schuck: ,Wenn man bedenkt, dass die Praxis, die durch den ,Heimspiel-Fonds ' gefördert wurde, inzwischen weit verbreitet ist und viel diskutiert wird, muss man wohl sagen, dass der Fonds sein Ziel erreicht hat: Es hat sich etwas bewegt. [...] Der Fonds hat aus meiner Sicht einen Ansatz aufgegriffen, den es schon gab, und ihn dann gefördert, also in die Breite gewirkt. Er war also Teil einer Entwicklung und wichtiger Anschub“ (Ebd.).
} 
Diese drei Beispiele zeigen exemplarisch, dass die Kulturstiftung des Bundes im Rahmen der Projekte des „Heimspielfonds“ durchaus als Impulsgeber bzw. Unterstützer von Transformationsprozessen wahrgenommen werden kann. Die Produktion neuer künstlerischer Formen und die von der Bundeskulturstiftung geschaffene finanzielle Möglichkeit zum „Ausprobieren“ führten unter anderem dazu, dass die Theater auch nach dem Abschluss der Stadtprojekte dem Partizipativen deutlich mehr Entwicklungsmöglichkeiten gegeben haben. Es kann also durchaus resultiert werden, dass der „Heimspielfonds“ eine künstlerische Transformation begleitet und unterstützt hat. Im Kontrast dazu sind ,angestoßene“ Transformationsprozesse auf organisationaler Ebene nicht klar zu verifizieren. Durch die Einführung von beispielsweise Bürgerbühnen haben sich Strukturen der Häuser sicherlich verändert, allerdings können diese nicht ausschließlich auf den durch den „Heimspielfonds“ ausgelösten künstlerischen Impuls zurückgeführt werden. Zuvorderst wurde von der Kulturstiftung des Bundes zunächst ausschließlich der Wunsch formuliert, dass durch den Erfolg des „Heimspielfonds“ „die Intendanten die Finanzierung solcher Formate vor dem Kulturausschuss und dem Abonnenten mit großer Überzeugung vertreten können“ (Völckers 2007). Bei den Intendant*innen scheinen die Impulse im Hinblick auf partizipative Theaterformen angekommen zu sein. So erklärt Intendant Ulrich Khuon:

„Partizipation verändert das, woran partizipiert wird. Die Institution muss folglich bereit sein, sich durch Partizipation auch selbst neu zu sehen und zu transformieren“ (Khuon 2019, S. 13).

Der Stellenwert von partizipativen Projekten bzw. Theaterformen mit Bezug zum Stadtraum hat sich also auch in den Leitungsebenen der Theater etabliert. Eine Entwicklung, welche partizipatives Theater und eine daraus entstehende Öffnung hin zur Stadtgesellschaft an den öffentlich getragenen Häusern in der Zukunft (weiter) stärkt.

\section{Literatur}

Badisches Staatstheater Karlsruhe. https://www.staatstheater.karlsruhe.de/programm/volkst heater/ueber/. Zugegriffen: 9. Juni 2020.

Baur, Detlev. 2011a. Auswärtsspiel oder Heimspiel? Deutsche Bühne 5, 30-33.

Baur, Detlev. 2011b. Profis und Laien im Dialog. Ein Gespräch mit Berit Schuck. Deutsche Bühne 2, 30-32.

Czirak, Adam. 2012. Partizipation der Blicke: Szenerien des Sehens und Gesehenwerdens in Theater und Performance. Bielefeld: Transcript. 
Czirak, Adam. 2014. Partizipation. In Metzler Lexikon Theatertheorie, Hrsg. E. FischerLichte, D. Kolesch, und M. Warstat, 242-248. Stuttgart: J.B. Metzler.

Deutscher Bühnenverein. 2003. Deutsche Bühne 4.

Deutscher Bühnenverein. 2004 bis 2011. Theaterstatistik.

Duden. https://www.duden.de/rechtschreibung/Partizipation. Zugegriffen: 9. Juni 2020.

Dreysse, M., und F. Malzacher. 2007. Experten des Alltags. Das Theater von Rimini Protokoll. Berlin: Alexander Verlag.

Hasselberg, Viola. 2014. Das Gegenmodell zur Dresdner Bürgerbühne: Theater als künstlerische Forschung am Theater Freiburg (2006-2013). In Die Bürgerbühne: Das Dresdner Modell, Hrsg. H. Kurzenberger, 159-171. Berlin: Alexander.

Khuon, Ulrich. 2019. Partizipation - Anstrengend, unausweichlich, transformierend. In Our Stage Festival Dokumentation, Hrsg. Staatsschauspiel Dresden und Miriam Tscholl, 1115. Dresden: Staatsschauspiel.

Klein, Armin. 2009. Kulturpolitik. Wiesbaden: Springer VS.

Kulturstiftung des Bundes. https://www.kulturstiftung-des-bundes.de/de/projekte/buehne_ und_bewegung/detail/heimspiel.html. Zugegriffen: 9. Juni 2020.

Kup, Johannes. 2019. Das Theater der Teilhabe. Berlin: Schibri.

Leber, Fabian. 2010. Kulturpolitik aus dem Kanzleramt. Marburg: Tectum.

Leucht, Sabine. 2006. Charmant unbrisant. Deutsche Bühne 7, 19.

Mundel, Barbara und J. Mackert. 2007. Erkundungen zum Stadttheater. Deutsche Bühne 7, $22-25$.

o. V. 2020. https://www.theaterpolitik.de/index.php/diskurse/das-stadttheater-reif-fuer-ref ormen/115-wem-gehoert-die-buehne-sechs-jahre-heimspiel-fonds-der-kulturstiftungdes-bundes. Zugegriffen: 9. Apr. 2020.

Scheurle, Christoph, M. Hinz, und N. Köhler. 2017. Partizipation: Teilhaben/teilnehmen. München: kopaed.

Schmidt, Thomas. 2017. Theater, Krise und Reform: Eine Kritik des deutschen Theatersystems. Wiesbaden: Springer VS.

Schmidt, Thomas. 2019. Macht und Struktur im Theater: Asymmetrien der Macht. Wiesbaden: Springer VS.

Schneider, W., und A. Eitzeroth. 2017. Partizipation als Programm: Wege ins Theater für Kinder und Jugendliche. Bielefeld: Transcript.

Sharifi, Azadeh. 2011. Theater für Alle? Partizipation von Postmigranten am Beispiel der Bühnen der Stadt Köln. Frankfurt a. M.: Lang.

Staatsschauspiel Dresden. 2020. https://www.staatsschauspiel-dresden.de/buergerbuehne/. Zugegriffen: 9. Juni 2020.

Völckers, Hortensia. 2007. Platzvorteil. Deutsche Bühne 7, 30/31.

Wagner, Bernd. 2004. Thema: Theaterdebatte. Essen: Klartext. 
Open Access Dieses Kapitel wird unter der Creative Commons Namensnennung 4.0 International Lizenz (http://creativecommons.org/licenses/by/4.0/deed.de) veröffentlicht, welche die Nutzung, Vervielfältigung, Bearbeitung, Verbreitung und Wiedergabe in jeglichem Medium und Format erlaubt, sofern Sie den/die ursprünglichen Autor(en) und die Quelle ordnungsgemäß nennen, einen Link zur Creative Commons Lizenz beifügen und angeben, ob Änderungen vorgenommen wurden.

Die in diesem Kapitel enthaltenen Bilder und sonstiges Drittmaterial unterliegen ebenfalls der genannten Creative Commons Lizenz, sofern sich aus der Abbildungslegende nichts anderes ergibt. Sofern das betreffende Material nicht unter der genannten Creative Commons Lizenz steht und die betreffende Handlung nicht nach gesetzlichen Vorschriften erlaubt ist, ist für die oben aufgeführten Weiterverwendungen des Materials die Einwilligung des jeweiligen Rechteinhabers einzuholen.

(c) (1) 